\title{
Retrospective Analysis of Long-Term Adherence to and Persistence with DPP-4 Inhibitors in US Adults with Type 2 Diabetes Mellitus
}

\author{
Amanda M. Farr · John J. Sheehan · Suellen M. Curkendall • \\ David M. Smith • Stephen S. Johnston • Iftekhar Kalsekar
}

To view enhanced content go to www.advancesintherapy.com

Received: October 23, 2014 / Published online: December 12, 2014

(c) The Author(s) 2014. This article is published with open access at Springerlink.com

\section{ABSTRACT}

Introduction: Patients with type 2 diabetes mellitus (T2DM) must remain adherent and persistent on antidiabetic medications to optimize clinical benefits. This analysis compared adherence and persistence among adults initiating dipeptidyl peptidase-4 inhibitors (DPP-4is), sulfonylureas (SUs), and thiazolidinediones (TZDs) and between patients initiating saxagliptin or sitagliptin, two DPP-4is. Methods: This retrospective cohort study utilized the US MarketScan ${ }^{\circledR}$ (Truven Health Analytics, Ann Arbor, MI, USA) Commercial and Medicare Supplemental health insurance claims databases. Adults aged $\geq 18$ years with T2DM who initiated a DPP-4i, SU, or TZD from

Electronic supplementary material The online version of this article (doi:10.1007/s12325-014-0171-3) contains supplementary material, which is available to authorized users.

A. M. Farr $(\bowtie)$. S. M. Curkendall · D. M. Smith . S. S. Johnston

Truven Health Analytics, 7700 Old Georgetown Road, 6th Floor, Bethesda, MD 20814, USA

e-mail: amanda.farr@truvenhealth.com

J. J. Sheehan · I. Kalsekar

AstraZeneca, 601 Office Center Drive, Suite 200,

Fort Washington, PA 19034, USA
January 1, 2009 to January 31, 2012 were included. Patients must have been continuously enrolled for $\geq 1$ year prior to and $\geq 1$ year following initiation. Adherence was measured using proportion of days covered (PDC), with $\mathrm{PDC} \geq 0.80$ considered adherent. Persistence was measured as time to discontinuation, defined as last day with drug prior to a $60+$ days gap in therapy. Multivariable logistic regression and Cox proportional hazards models compared the outcomes between cohorts, controlling for baseline differences.

Results: The sample included 238,372 patients (61,399 DPP-4i, 134,961 SU, 42,012 TZD). During 1-year follow-up, $47.3 \%$ of DPP-4i initiators, $41.2 \%$ of SU initiators, and $36.7 \%$ of TZD initiators were adherent. Adjusted odds of adherence were significantly greater among DPP-4i initiators than SU (adjusted odds ratio $[\mathrm{AOR}]=1.678, P<0.001$ ) and TZD initiators $(\mathrm{AOR}=1.605, \quad P<0.001) . \quad$ During $\quad 1$-year follow-up, $55.0 \%$ of DPP-4i initiators, $47.8 \%$ of SU initiators, and $42.9 \%$ of TZD initiators did not discontinue therapy. Adjusted hazards of discontinuation were significantly greater for SU (adjusted hazard ratio $[\mathrm{AHR}]=1.390$, 
$P<0.001)$ and TZD initiators $(\mathrm{AHR}=1.402$, $P<0.001)$ compared with DPP-4i initiators. Saxagliptin initiators had significantly better adherence $(\mathrm{AOR}=1.213, P<0.001$ ) compared with sitagliptin initiators, and sitagliptin initiators had significantly greater hazard of discontinuation $\quad(\mathrm{AHR}=1.159, \quad P<0.001)$. Results were similar over a 2-year follow-up.

Conclusions: US adults with T2DM who initiated DPP-4i therapy, particularly saxagliptin, had significantly better adherence and persistence compared with patients who initiated SUs or TZDs.

Keywords: Adherence; Dipeptidyl peptidase-4 inhibitors; Persistence; Type 2 diabetes mellitus

\section{INTRODUCTION}

In the US, an estimated 26 million people have diabetes, approximately $8 \%$ of the entire population [1]. Diabetic complications are an important medical and public health concern, as diabetes is associated with kidney failure, limb amputation, blindness, heart disease, and stroke [1]. Appropriate disease management, primarily maintenance of glycated hemoglobin (HbA1c) levels below 7\% can decrease the risk of microvascular complications [2]. Current treatments for type 2 diabetes mellitus (T2DM) include lifestyle and dietary modifications, oral antidiabetic medications, non-insulin injectables, and insulin [2, 3]. For patients with mild hyperglycemia, lifestyle change and monotherapy with metformin or an acceptable alternative, such as a dipeptidyl peptidase-4 inhibitor (DPP-4i), are recommended [3]. Sulfonylureas (SUs) and thiazolidinediones (TZDs) can also be prescribed, although it has been suggested that the additional risks associated with these drug classes should be considered [3]. The American Diabetes Association, European Association for the Study of Diabetes, and the American Association of Clinical Endocrinologists recommend that patients who do not reach HbA1c targets should be prescribed combination therapy, followed by insulin initiation if necessary $[2,3]$.

To optimize the clinical benefits of antidiabetic pharmacotherapy, patients must remain adherent to and persistent with their prescribed medications. The importance of adherence to antidiabetic medications has been recognized by Medicare and appears as a Medicare Part D plan quality measure [4]. Increased adherence to medications is associated with a decrease in HbA1c [5-8], lower odds of mortality [7], fewer all-cause hospitalizations [7], and lower healthcare expenditures [9-12]. These improvements in outcomes may translate into substantial benefit to the healthcare system, averting a projected 699,000 emergency room visits and 341,000 hospitalizations per year, resulting in a savings of nearly $\$ 5$ billion [13]. Despite the noted impact of good adherence, a systematic review found a wide range of adherence rates across retrospective studies, with rates ranging from $36 \%$ to $93 \%$ [14]. A second review found that when combining study results, only $58 \%$ of patients were considered adherent [15]. To date, the majority of interventions to improve adherence to diabetes medications have focused on education and one-on-one counseling [16].

Several factors associated with adherence to antidiabetic medications have been identified in the literature. Younger age, female gender, residence in the Southern region of the US, and high cost-sharing have been associated with lower adherence in multiple analyses $[10,17$, 18]. Additional factors include depression, 
polytherapy, and twice-daily regimens [14]. Analyses have also compared patients initiating specific non-insulin antidiabetic drugs and drug classes in terms of adherence and persistence, including metformin, SUs, glucagon-like peptide-1 receptor agonists (GLP1RAs), TZDs, and DPP-4is [10, 17, 19, 20].

To our knowledge, no US-based analyses comparing adherence to and persistence with DPP-4is as a medication class with other classes of antidiabetic medications have been reported. Therefore, the objective of this analysis was to compare adherence and persistence among patients initiating DPP-4is, SUs, and TZDs over 1- and 2-year follow-up periods. Additional objectives were to compare adherence and persistence between patients initiating saxagliptin and sitagliptin and to describe treatment patterns following initiation.

\section{METHODS}

\section{Data Source}

This retrospective, observational cohort study used administrative claims data from the Truven Health MarketScan ${ }^{\circledR}$ (Truven Health Analytics, Ann Arbor, MI, USA) Commercial and Medicare Supplemental databases. The databases contain inpatient medical, outpatient medical, and outpatient prescription administrative claims data and enrollment records of individuals with employer-sponsored primary or Medicare supplemental insurance. The Commercial and Medicare Supplemental Databases have been utilized in numerous published analyses [21]. The data were previously collected, deidentified, and compliant with the Health Insurance Portability and Accountability Act privacy regulations. Therefore, Institutional
Review Board approval and written informed consent were not required for this study.

\section{Patient Selection}

Patients with at least one outpatient pharmacy claim with a National Drug Code (NDC) for a DPP-4i, SU, or TZD from January 1, 2009 to January 31, 2012, were selected. A rolling selection method was used, whereby each drug claim was evaluated on the criteria described below and the earliest claim meeting all criteria was selected as the index date and the medication class (DPP-4i, SU, or TZD) was considered the index drug class. Patients must have had at least 28 days of continuous supply of the index drug class and have been at least 18 years old on the index date. All patients were required to have at least 365 days of continuous enrollment with medical and pharmacy benefits prior to the index date (pre-period) and at least 365 days of enrollment following the index date (1-year follow-up period). A subset of patients was required to have 730 days of post-index enrollment (2-year follow-up period). To capture initiators only, patients were required to have no claims for the index drug class in the preperiod. Lastly, patients were required to have at least one non-diagnostic medical claim with a T2DM diagnosis (International Classification of Diseases, Ninth Revision, Clinical Modification [ICD-9-CM] 250.x0, 250.x2) during the study period. Patients with medical claims with diagnoses of type 1 diabetes (ICD-9-CM 250.x1, 250.x3) or gestational diabetes (ICD-9-CM $648.8 x$ ) or with multiple index drug classes on index date were excluded from analysis.

\section{Outcome Variables}

The primary outcomes were adherence to and persistence with the index drug class. Both 
measures were calculated using the service date and days' supply fields existing on outpatient pharmacy claims for DPP-4is, SUs, and TZDs identified by NDC codes. Adherence was measured as proportion of days covered (PDC), calculated by taking the number of days a patient had the index drug class on hand during the 1- or 2-year follow-up based on the days' supply field on pharmacy claims divided by follow-up time (365 or 730 days). Days' supply for early refills was appended to the end of days' supply of the previous prescription. Patients with a PDC $\geq 0.80$ were considered adherent. Persistence was defined as the time from index date to last day with index drug class on hand prior to a gap of $>60$ consecutive days without index drug class [22]. A cut-point of 60 days was utilized as a conservative definition of discontinuation, as patients with gaps of up to 60 days were considered persistent. Switching within drug class was allowed for the drug class comparison. When comparing adherence and persistence outcomes between patients initiating saxagliptin and sitagliptin, PDC and time to discontinuation were calculated at the drug level, rather than the drug class level.

Patients were classified into the following mutually exclusive groups based on first event after index date during the 1-year follow-up period: remained on index drug class with no augmentation, augmentation with additional drug class, discontinuation of index drug class and switch to a new drug class, discontinuation of study drug class and continuation on other medication classes with no switch, and discontinuation of all antidiabetic medications. An augmentation was defined as the addition of a medication class not part of the initial regimen that overlapped with the index drug class for $>30$ days. A switch was defined as the discontinuation of index drug class and the addition of medication class not in the initial regimen prior to discontinuation with overlap $\leq 30$ days or following discontinuation. Discontinuation was measured at the drug class level for all cohort comparisons.

\section{Independent Variables}

The primary independent variable of interest was index drug class: DPP-4i, SU, or TZD. When comparing within the DPP-4i medication class, the primary predictor was index drug: saxagliptin or sitagliptin. A third DPP-4i, linagliptin, was not compared within the DPP4i-specific analysis because few linagliptin initiators had 1 year of follow-up, and no linagliptin initiators had 2 years of follow-up in the claims data. A fourth DPP-4i, alogliptin, was not available in the US during the patient selection period.

Demographic, clinical, and cost and utilization characteristics were measured to describe the study sample and to control for potential confounding in multivariable models. Demographic measures included: sex, age, geographic region, urbanicity, insurance plan type, primary payer, presence of capitated services, and year of index date. Clinical characteristics were measured during the preperiod and included use of study drugs other than index drug class, and metformin and insulin use based on pharmacy claims, overall health captured by the Deyo Charlson Comorbidity Index (CCI) [23] and the number of unique ICD-9-CM codes appearing on a patient's medical claims, diagnosis of macrovascular disease (acute myocardial infarction, other ischemic heart disease, congestive heart failure, cerebrovascular accident, or peripheral vascular disease) and microvascular disease (diabetic peripheral 
neuropathy, diabetic retinopathy, leg and foot amputation, or nephropathy), and diagnosis or procedure indicative of renal impairment. Pregnancy during the follow-up period was captured, as it may affect diabetes treatment. Cost and utilization covariates captured during the pre-period were evidence of an endocrinologist visit and cardiologist visit, total healthcare expenditures, and diabetes prescription expenditures.

Several characteristics of the index drug prescription claim were also captured: the days' supply, mail-order status, fixed-dose combination with metformin, cost-sharing index, which was the average patient out-ofpocket cost for a 30-day supply, calculated annually for each study drug for each insurance plan in the database, and index regimen [24]. A patient's initial regimen was defined as the index drug class plus any concomitant use of additional antidiabetic medication classes (alpha-glucosidase inhibitors, amylin analogs, dopamine receptor agonists, GLP-1RAs, insulin, meglitinides, and metformin). Claims with service dates occurring 60 days prior to the index date to 45 days after were evaluated. A medication class was considered part of the initial regimen if (a) the patient received a prescription for the drug class within 60 days before index date and received a second prescription for that drug class within 45 days after index date or (b) the patient received a prescription for the drug within 60 days before index date or 45 days after index date and had $\geq 30$ days' overlap between index drug class and additional drug class between index date and index date +45 days. This definition was purposely conservative so as not to misclassify switches as part of a patient's initial regimen. Patients with no additional medication classes meeting this definition were considered to be on monotherapy.

\section{Analysis}

In descriptive analyses, pairwise comparisons using $t$ tests and Chi-squared tests were conducted comparing SU initiators and TZD initiators with DPP-4i initiators and saxagliptin initiators with sitagliptin initiators. Multivariable logistic regression models were fit to compare the odds of being adherent, and multivariable Cox proportional hazards models were fit to compare time to discontinuation (persistence) between index drug classes and index drugs. To determine which covariates were included in the multivariable models, descriptive statistics and statistical tests were evaluated to identify characteristics that differed between cohorts and also may be associated with adherence or persistence (i.e., were potential confounders). Models controlled for the following variables: age, sex, presence of capitated services, payer, region, urbanicity, plan type, indicator for fixed-dose metformin index drug, indicator for index drug filled via mail order, index drug regimen (monotherapy, index drug plus additional non-insulin antidiabetic drugs, index drug plus insulin), baseline total expenditures and diabetes prescription expenditures, index diabetes medication class cost-sharing, baseline endocrinologist and cardiologist visits, baseline renal impairment, baseline macrovascular and microvascular disease, pregnancy during follow-up, baseline number of unique three-digit ICD-9-CM diagnoses and Charlson Comorbidity Index. Sensitivity analyses of monotherapy and non-mail-order patients were also conducted, as patients initiating monotherapy have the simplest treatment profile and those with an initial prescription filled via mail order may have inflated persistence due to larger days' supply on the index claim and automatic refill. $P$ values 
were compared with an a priori alpha 0.05 to determine statistical significance. Analyses were conducted with SAS version 9.3 (SAS Institute Inc. Cary, NC, USA).

\section{RESULTS}

\section{Patient Selection}

Patient attrition is presented in Fig. 1. There were $1,788,908$ patients identified from the databases with at least one prescription claim for a DPP-4i, SU, or TZD from January 1, 2009 to January 31, 2012. After applying additional inclusion and exclusion criteria, the final sample consisted of 238,372 patients; 61,399 patients initiated a DPP-4i, 134,961 initiated an SU, and 42,012 initiated a TZD. Within DPP-4i initiators, 11,219 patients initiated saxagliptin and 49,400 initiated sitagliptin. A total of 134,444 patients had 2 years of follow-up data.

\section{Descriptive Analysis}

Demographic, clinical, and treatment characteristics are presented in Tables 1 and 2 . The average age of patients in the sample was 56.7 years (standard deviation [SD] 12.2), and $54.3 \%$ were men. The majority of patients were insured through a commercial plan (77.7\%). Approximately, one-fifth (20.3\%) had evidence of macrovascular disease in the pre-period. Metformin use was common in the pre-period $(55.0 \%)$, whereas insulin use was less common (6.7\%). There were significant differences in baseline characteristics among the three drug class cohorts. DPP-4i initiators were younger on average than SU initiators but older than TZD initiators, and a greater proportion were women than both SU and TZD initiators. The proportion of patients using metformin in the pre-period was higher among DPP-4i initiators, and a greater proportion of patients initiated a study drug that was part of a fixed-dose combination with metformin. A mail-order index prescription was more common among DPP-4i initiators, whereas initiating monotherapy was less common. Significant differences were also found between the saxagliptin and sitagliptin initiators. On average, saxagliptin initiators were younger and more commonly insured through commercial plans. The prevalence of renal impairment, macrovascular disease, and microvascular disease was lower among saxagliptin initiators. More saxagliptin initiators initiated monotherapy compared with sitagliptin initiators.

Figure 2 presents treatment patterns following index drug class initiation. Among all study cohorts, augmentation was the least common event, although augmentation was more common among DPP-4 $\mathrm{i}$ initiators. A significantly larger proportion of DPP-4i initiators remained on index drug class compared with SU and TZD initiators. Among DPP-4i initiators, the proportion of patients remaining on DPP-4is was significantly larger among the saxagliptin initiators. Overall, 20.6\% of the study sample discontinued all antidiabetic medications for $>60$ days without first switching or augmenting. Discontinuing all antidiabetic medications was least common among DPP-4i initiators because, as noted above, remaining on initial regimen or augmenting was more common. In the DPP4i-specific comparison, discontinuing all antidiabetic medications was less common among saxagliptin initiators. Within the first year, over $40 \%$ of SU and TZD initiators stopped refilling their index drug class, either discontinuing all antidiabetic medications or 


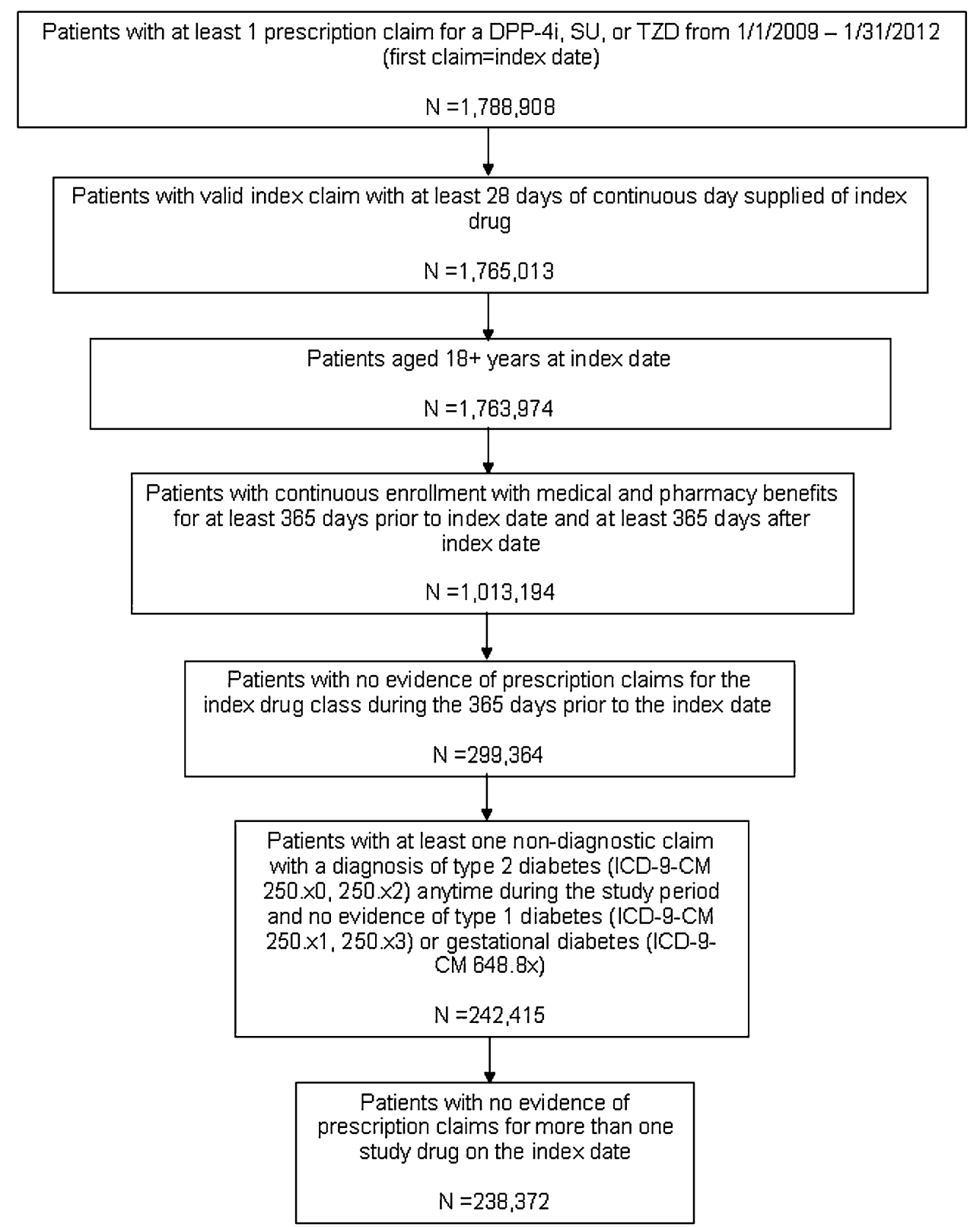

Fig. 1 Patient attrition. DPP-4i Dipeptidyl peptidase-4 inhibitor, ICD-9-CM International Classification of Diseases, Ninth Revision, Clinical Modification, SU Sulfonylurea, TZD Thiazolidinedione

continuing on the other medications in their initial regimen.

Unadjusted adherence and persistence results are presented in Table 3. Over the 1-year follow-up period, a significantly greater proportion of DPP-4i initiators were adherent to index drug class than SU and TZD initiators.
This relationship was consistent over the 2-year follow-up period and in the monotherapy and the non-mail-order sub-samples. Significantly more DPP-4i initiators were persistent with index drug class during the 1-year follow-up period than SU initiators and TZD initiators. As with adherence, this was true over the 2-year 


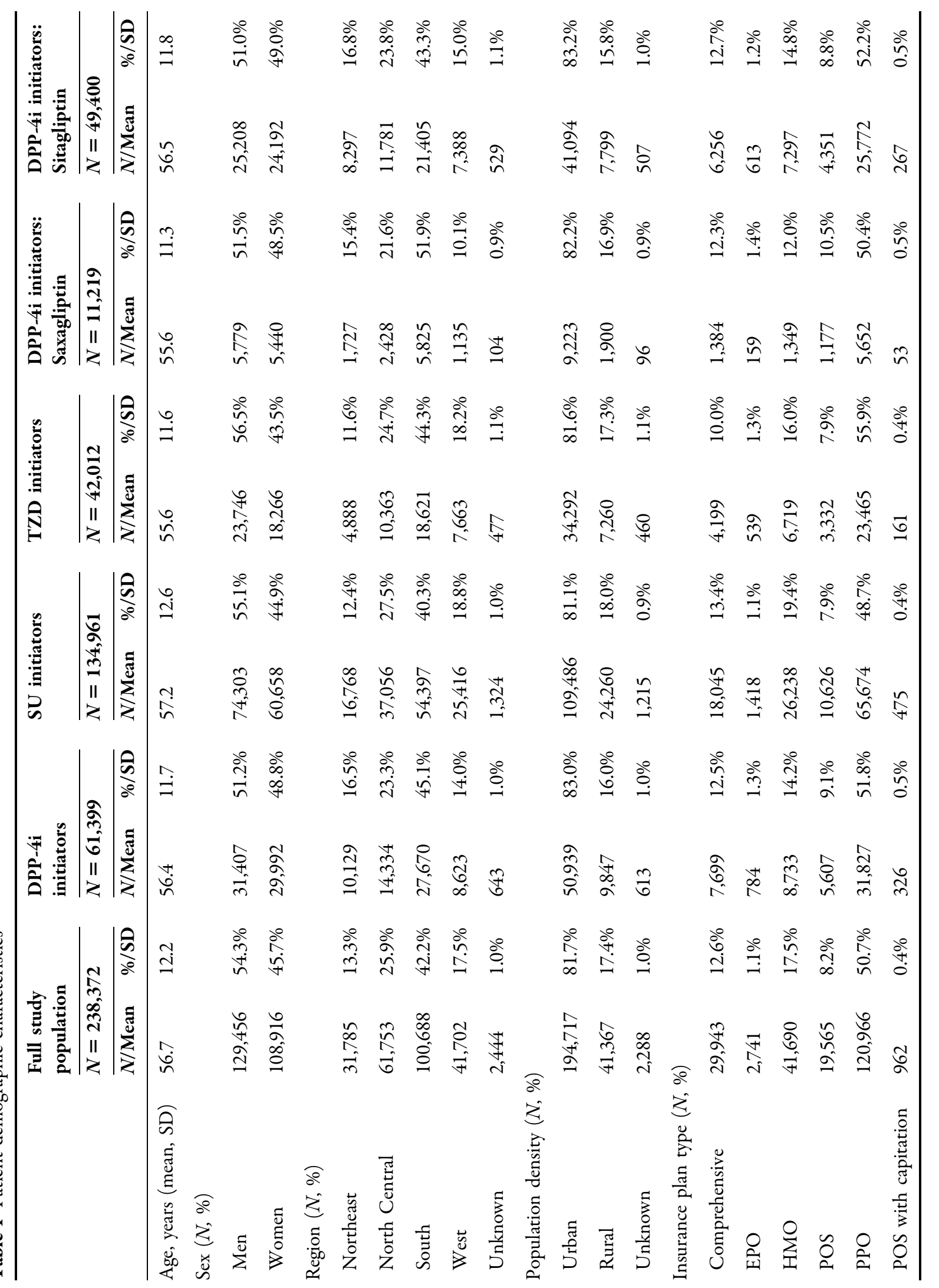




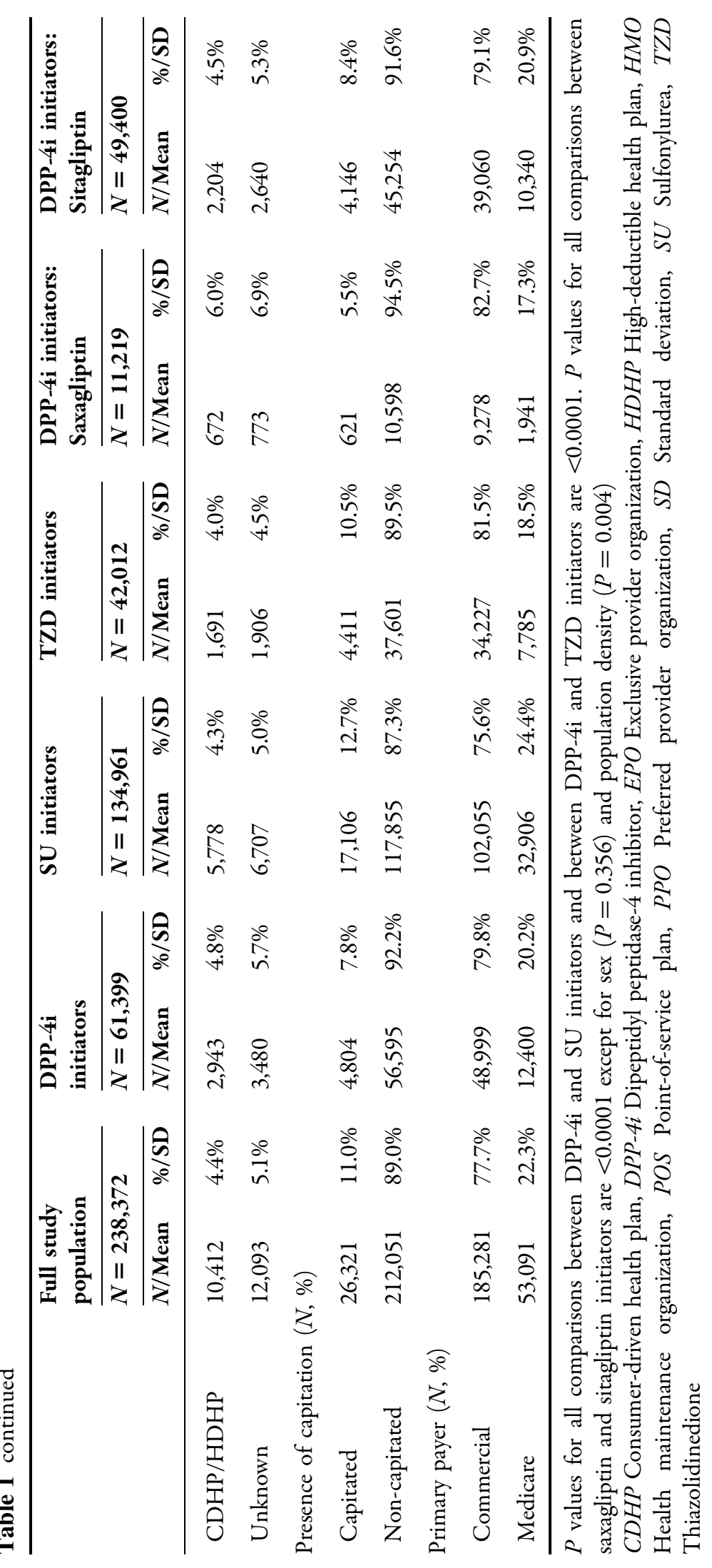




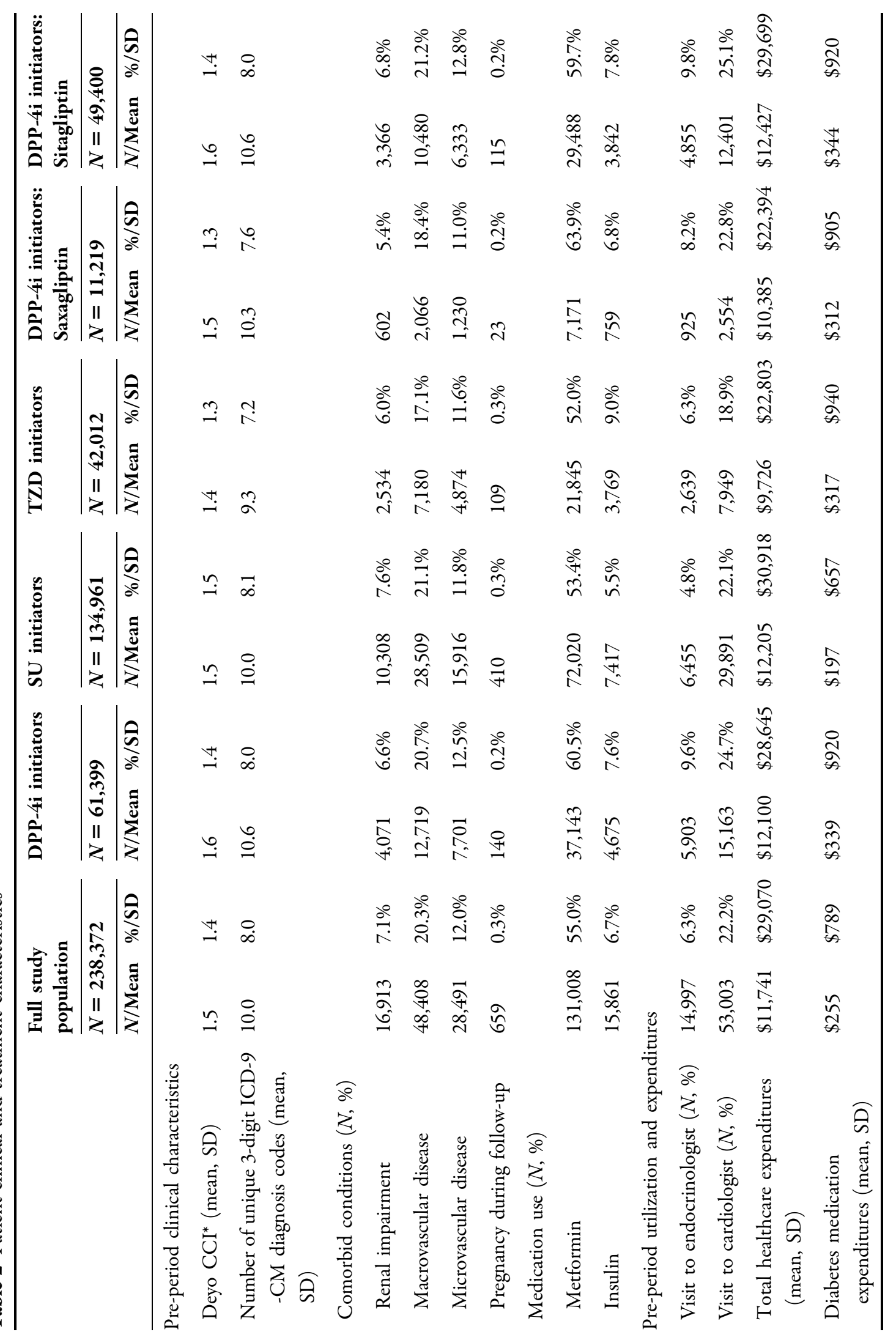




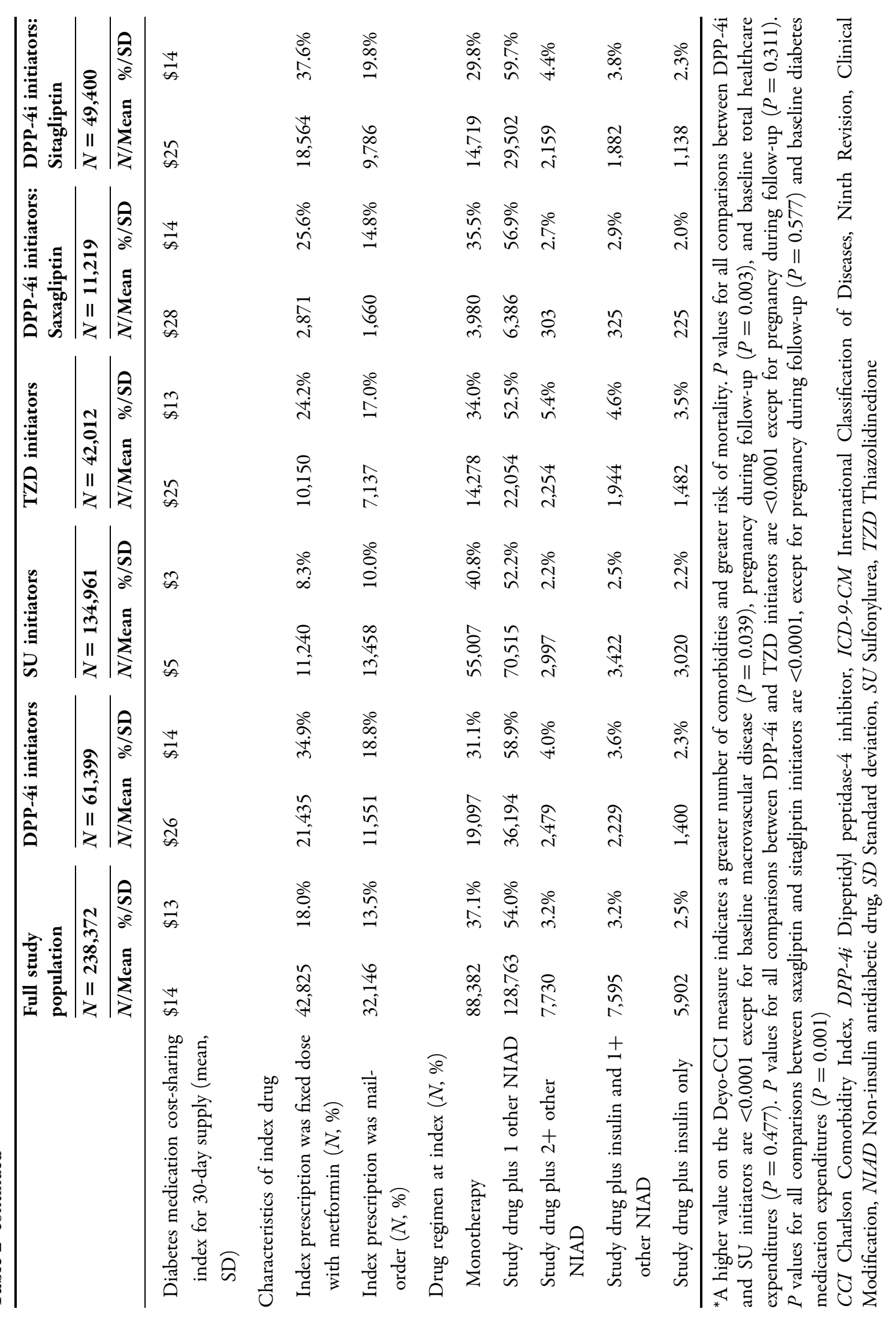


First Treatment Event During 1-Year Follow-Up

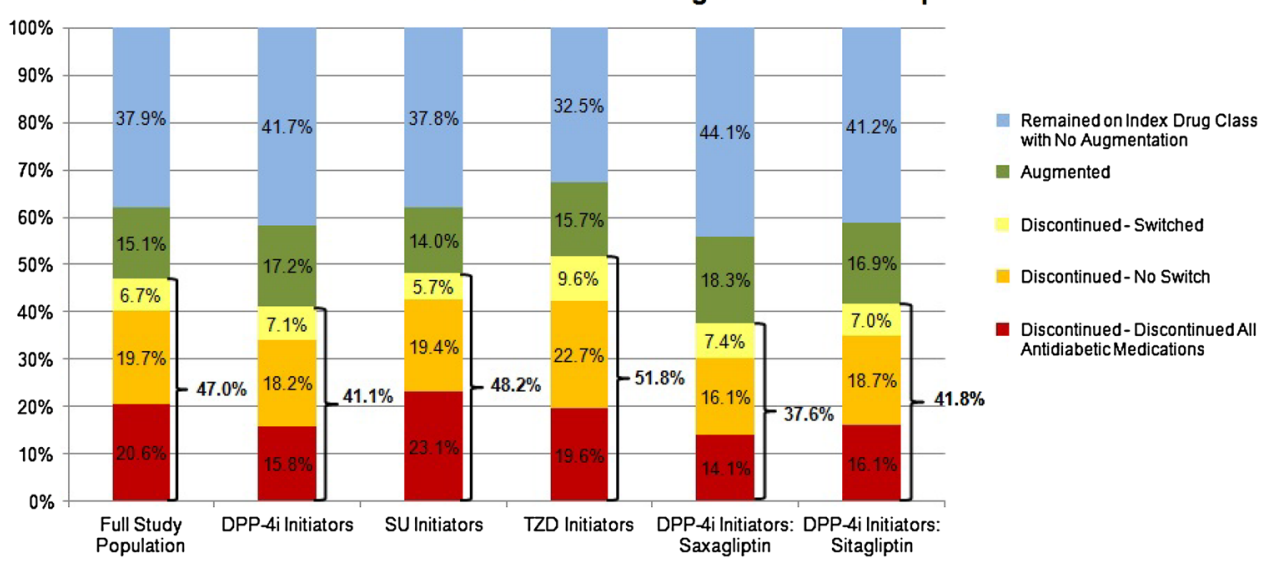

Fig. 2 First treatment event after index drug class/index drug initiation. $P$ values for comparing DPP-4i and SU initiators, DPP-4i and TZD initiators, and saxagliptin and sitagliptin initiators were $<0.001$. Discontinuation

follow-up period and in both sub-samples. Among DPP-4i initiators, patients initiating saxagliptin had better unadjusted persistence to index drug than patients initiating sitagliptin overall and in sub-samples. There were significant differences in adherence between the two cohorts in all samples except monotherapy patients. Descriptively, a smaller proportion of linagliptin patients were adherent (43.5\%) compared with saxagliptin patients (49.1\%) and sitagliptin patients (46.1\%) and a smaller proportion of linagliptin patients were persistent (50.9\% vs. $56.6 \%$ for saxagliptin and $53.6 \%$ for sitagliptin) during the first year of follow-up. No statistical comparisons were conducted due to small sample size.

\section{Multivariable Analysis}

Figure 3 presents adherence results. In adjusted logistic regression models, patients initiating DPP-4is had significantly greater odds of being adherent than patients initiating an SU or TZD. During the 1-year follow-up period, the adjusted odds of being adherent among DPP-4i
( $>60$ days without drug on hand) was measured at drug class level for all cohorts. DPP-4i Dipeptidyl peptidase-4 inhibitor, $S U$ Sulfonylurea, TZD Thiazolidinedione

initiators was over 1.6 times the odds of being adherent among SU initiators (adjusted odds ratio $[\mathrm{AOR}]=1.678,95 \%$ confidence interval $[C I]=1.631, \quad 1.727)$ and TZD initiators $(\mathrm{AOR}=1.605,95 \% \mathrm{CI}=1.563,1.647)$. Among DPP-4i initiators, patients initiating saxagliptin had significantly greater odds of being adherent than patients initiating sitagliptin $(\mathrm{AOR}=1.213,95 \% \mathrm{CI}=1.161,1.266)$. Results for the 2-year follow-up period and for subsets of monotherapy and non-mail-order patients were of similar direction, magnitude, and significance.

Figures 4 and 5 present adjusted curves for time to discontinuation. In adjusted Cox proportional hazards models, the adjusted hazard of discontinuation was approximately $40 \%$ higher among SU initiators (adjusted hazard ratio $[\mathrm{AHR}]=1.390,95 \% \mathrm{CI}=1.363$, 1.418) and TZD initiators (AHR $=1.402,95 \%$ $\mathrm{CI}=1.377, \quad 1.427) \quad$ compared with DPP-4i initiators during 1-year follow-up. Over the same time period, patients who initiated sitagliptin were significantly more likely to discontinue than those who initiated 


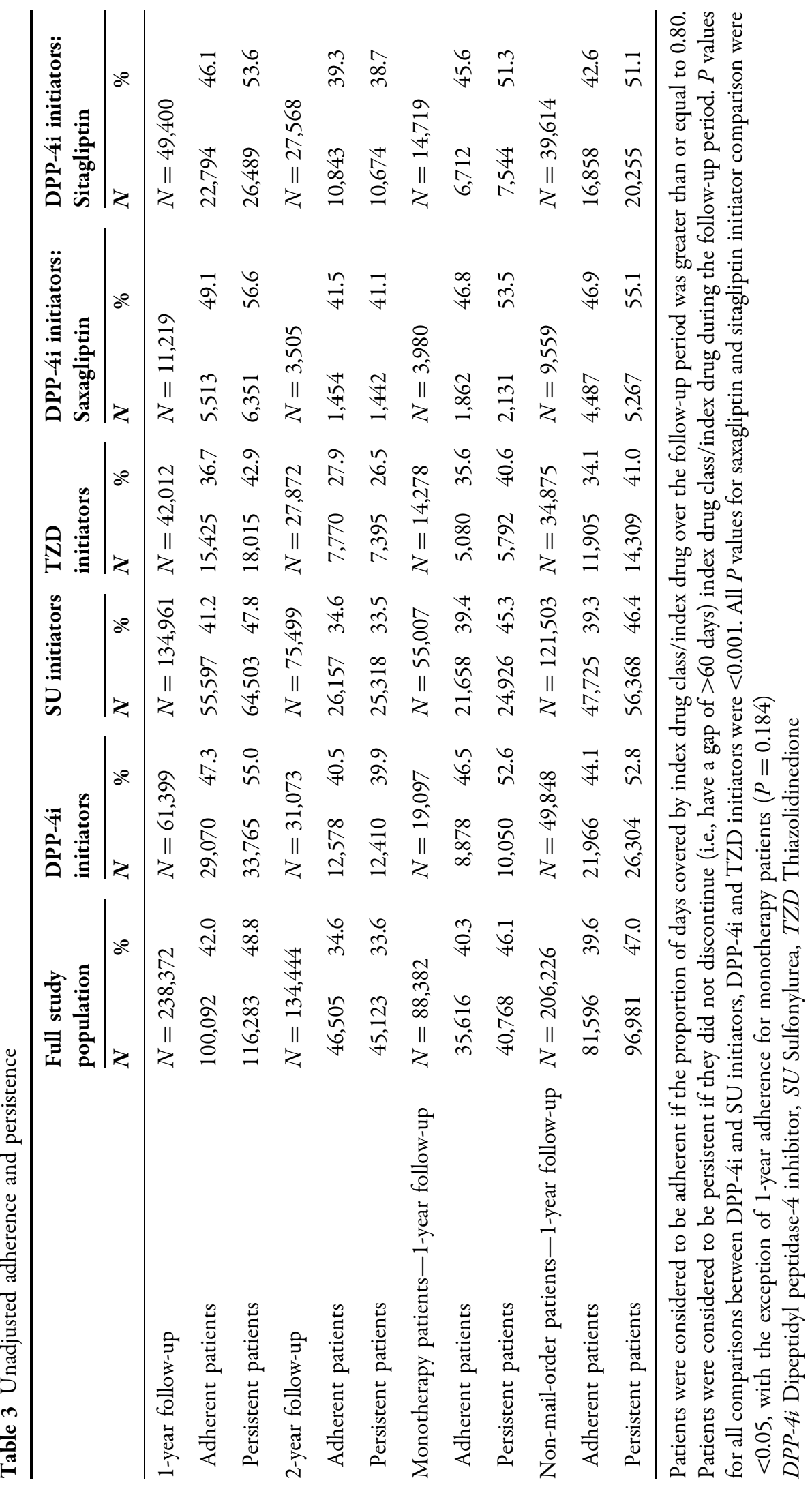




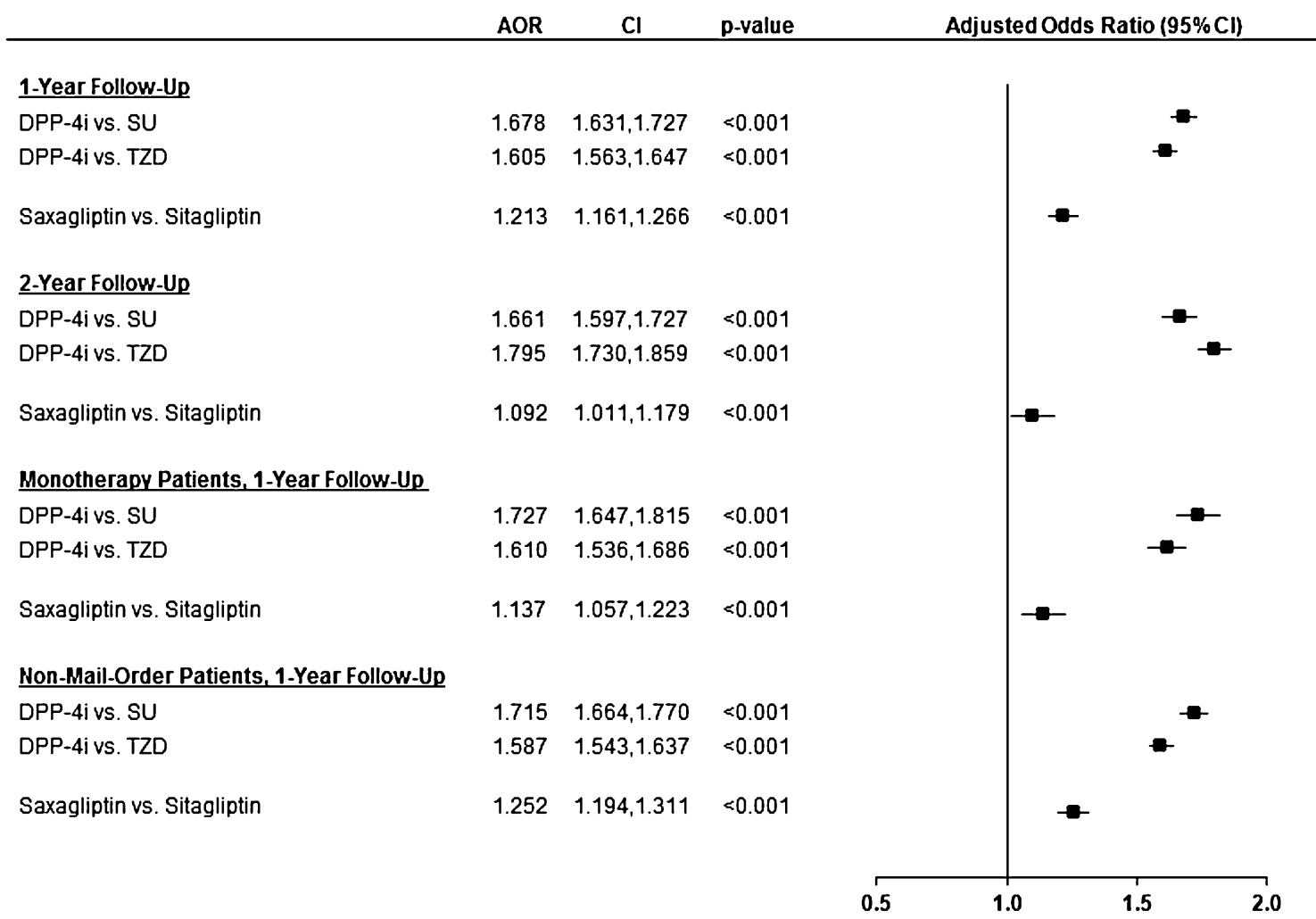

Fig. 3 Adjusted odds ratio for being adherent $(P D C \geq 0.80)$ by index drug class and index drug within DPP-4i cohort for 1- and 2-year follow-up among full study sample and sub-samples of monotherapy and non-mailorder patients. Models controlled for age, sex, presence of capitated services, payer, region, urbanicity, plan type, indicator for fixed-dose metformin index drug, indicator for index drug filled via mail order, index drug regimen (monotherapy, index drug plus additional non-insulin antidiabetic drugs, index drug plus insulin), baseline total

saxagliptin $\quad(\mathrm{AHR}=1.159, \quad 95 \% \quad \mathrm{CI}=1.122$, 1.195). As with adherence, these associations held over the 2-year follow-up period and within the two patient subgroups.

\section{DISCUSSION}

In this US administrative claims-based analysis of adults with T2DM, patients who initiated a DPP-4i had significantly better adherence to and persistence with index drug class compared with patients who initiated an SU expenditures and diabetes prescription expenditures, index diabetes medication class cost-sharing, baseline endocrinologist and cardiologist visits, baseline renal impairment, baseline macrovascular and microvascular disease, pregnancy during follow-up, baseline number of unique 3-digit ICD-9 diagnoses and Charlson Comorbidity Index. AOR Adjusted odds ratio, CI Confidence interval, DPP-4i Dipeptidyl peptidase-4 inhibitor, $P D C$ Proportion of days covered, $S U$ Sulfonylurea, TZD Thiazolidinedione

or TZD. Among DPP-4i initiators, saxagliptin patients had significantly better adherence and persistence than sitagliptin patients. These associations were observed over a 1-year follow-up period, a 2-year follow-up period, and within sub-samples of patients initiating monotherapy and filling index prescription through a non-mail-order method.

Patients may discontinue their oral antidiabetic medication following the onset of adverse events particular to their medication, 


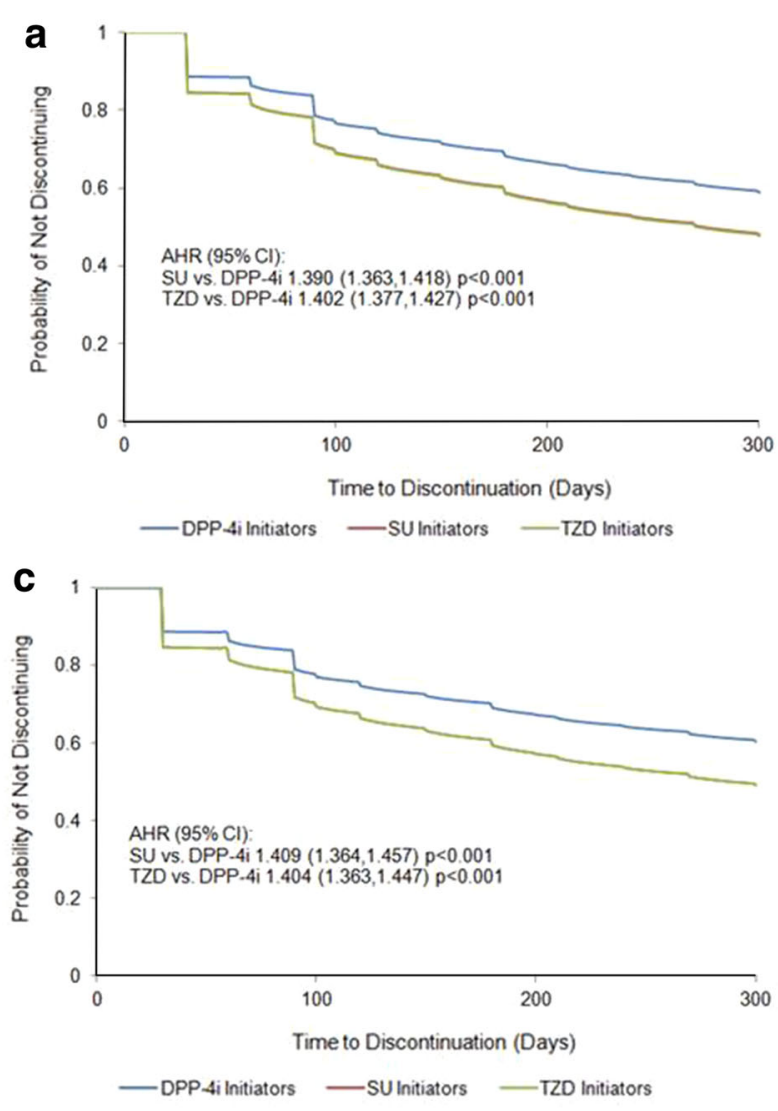

Fig. 4 Adjusted curves for time to discontinuation by index drug class for 1- and 2-year follow-up and within subsamples of monotherapy and non-mail-order patients for 1-year follow-up. a Adjusted curves and hazard ratios for discontinuation of index drug class over 1-year follow-up period. b Adjusted curves and hazard ratios for discontinuation of index drug class over 2-year follow-up period. c Adjusted curves and hazard ratios for discontinuation of index drug class over 1-year follow-up period among monotherapy patients. $\mathbf{d}$ Adjusted curves and hazard ratios for discontinuation of index drug class over 1-year follow-up period among non-mail-order patients. Models controlled for age, sex, presence of capitated services, payer, region, urbanicity, plan type, indicator for

such as hypoglycemia while on an SU $[25,26]$. Additionally, clinicians may discontinue a medication when the patient develops a comorbidity that places the patient at greater risk for an adverse event with a particular medication, for example, a patient at increasing risk for fractures on a TZD [27].
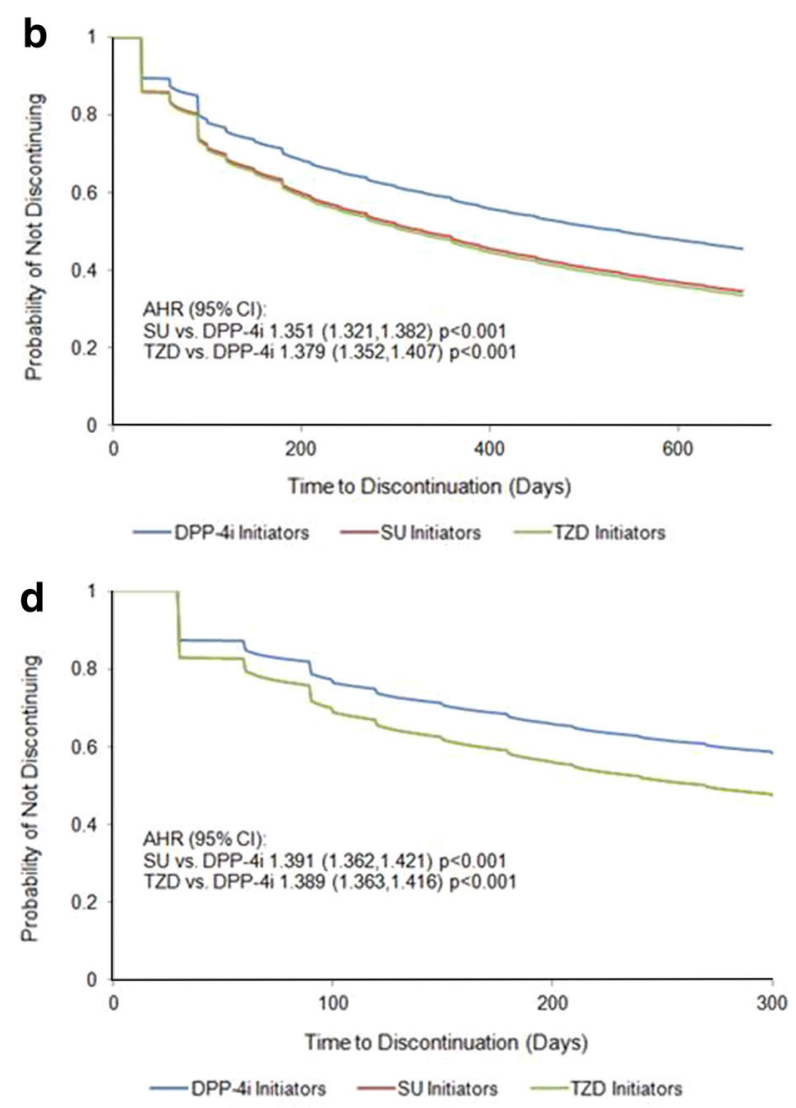

fixed-dose metformin index drug, indicator for index drug filled via mail order, index drug regimen (monotherapy, index drug plus additional non-insulin antidiabetic drugs, index drug plus insulin), baseline total expenditures and diabetes prescription expenditures, index diabetes medication class cost-sharing, baseline endocrinologist and cardiologist visits, baseline renal impairment, baseline macrovascular and microvascular disease, pregnancy during follow-up, baseline number of unique 3-digit ICD-9 diagnoses and Charlson Comorbidity Index. $A H R$ Adjusted hazard ratio, CI Confidence interval, DPP-4i Dipeptidyl peptidase-4 inhibitor, $S U$ Sulfonylurea, $T Z D$ thiazolidinedione

Potential explanations for the observed association between initiation of DPP-4is and better adherence and persistence include a better tolerability profile compared with SUs, which are associated with weight gain and hypoglycemia, and TZDs, which are associated with weight gain and bone fracture [3]. 

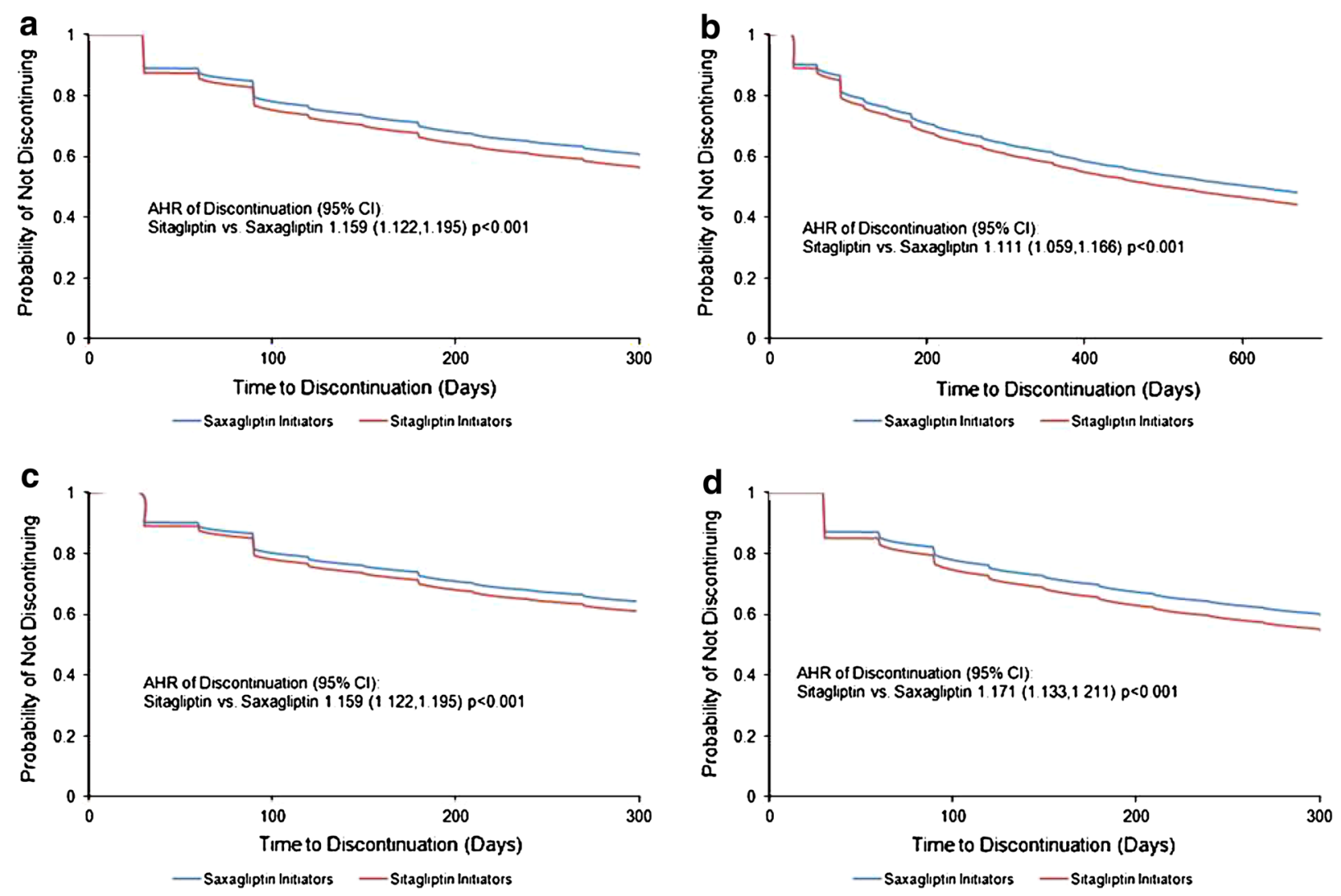

Fig. 5 Adjusted curves for time to discontinuation by index drug for 1- and 2-year follow-up and within subsamples of monotherapy and non-mail-order patients for 1-year follow-up. a Adjusted curves and hazard ratios for discontinuation of index drug over 1-year follow-up period. b Adjusted curves and hazard ratios for discontinuation of index drug over 2-year follow-up period. c Adjusted curves and hazard ratios for discontinuation of index drug over 1-year follow-up period among monotherapy patients. d Adjusted curves and hazard ratios for discontinuation of index drug over 1-year follow-up period among nonmail-order patients. Models controlled for age, sex, presence of capitated services, payer, region, urbanicity, plan type,

Differences noted between saxagliptin and sitagliptin may be due in part to a one-step dose adjustment for patients with renal impairment for saxagliptin, compared with a two-step dose adjustment for sitagliptin [28, 29]. However, more research utilizing additional types of data sources is needed to specifically test these hypotheses for the

indicator for fixed-dose metformin index drug, indicator for index drug filled via mail order in monotherapy samples, index drug regimen (monotherapy, index drug plus additional non-insulin antidiabetic drugs, index drug plus insulin) in non-mail-order samples, baseline total expenditures and diabetes prescription expenditures, index diabetes medication class cost-sharing, baseline endocrinologist and cardiologist visits, baseline renal impairment, baseline macrovascular and microvascular disease, pregnancy during follow-up, baseline number of unique 3-digit ICD-9 diagnoses and Charlson Comorbidity Index. $A H R$ Adjusted hazard ratio, $C I$ Confidence interval, $D P P-4 i$ Dipeptidyl peptidase-4 inhibitor

differences in adherence and persistence between drug classes and DPP-4i-specific drugs.

An analysis conducted by Rathmann et al. [20] comparing DPP-4is and SUs reported results consistent with the findings presented here. Utilizing data from general practices in Germany, researchers found that the risk of discontinuation was higher for DPP-4i initiators 
in the first 3 months and then fell below that of SU initiators for the remainder of follow-up, a maximum of 2 years [20]. Using US health insurance claims, Curkendall et al. [10] evaluated adherence and persistence over 1-year follow-up between patients initiating saxagliptin and patients initiating an SU, TZD, or GLP-1RA. These investigators found that patients initiating saxagliptin had greater odds of being adherent and lower risk of discontinuation than patients initiating medications within the other three drug classes [10]. No analyses over a 2-year followup or within monotherapy or non-mail-order subgroups were conducted [10]. Beyond adherence and persistence, Rathmann et al. [20] found that compared with SUs, initiating a DPP-4i was associated with lower risk of hypoglycemia and newly diagnosed macrovascular outcomes, including coronary heart disease, myocardial infarction, and stroke.

This study has limitations that should be recognized. Administrative claims data are not collected for research purposes, and the diagnostic coding on administrative claims is recorded by healthcare providers to support reimbursement. Consequently, misclassification of certain study variables, comorbid conditions in particular, may have occurred. Similarly, unmeasured confounding may exist, as clinical variables such as HbA1c level and duration of disease, socioeconomic characteristics such as income and education, and other factors such as provider characteristics were not available for inclusion in the claims databases. As this was a retrospective, observational analysis, causal inferences should be drawn with caution. Calculations of adherence and persistence were based on the service date and days' supply from outpatient prescription drug claims and assume that patients took the medication as directed. It cannot be determined whether patients skipped doses or discontinued the medication prior to the end of the current days' supply. Any prescriptions obtained without generating a health insurance claim were not captured in this analysis. The reason for discontinuation (e.g., adverse events, failure to achieve treatment goals, etc.) is not available in claims databases. Lastly, the MarketScan Commercial and Medicare Supplemental databases contain data only on patients covered through employers, private commercial insurance, or employer-sponsored supplemental Medicare. Thus, findings from the study may not be generalizable to the whole US Medicare population, Medicaid population, or the uninsured.

\section{CONCLUSIONS}

Patients initiating DPP-4is were more adherent to and persistent with the index drug class than patients initiating SUs or TZDs. These findings were robust to sensitivity analyses. Similarly, patients initiating saxagliptin were more likely to be adherent and persistent than patients initiating sitagliptin. These associations between DPP-4is and adherence and persistence support the use of DPP-4i medications, specifically saxagliptin, in management of T2DM, as adherence and persistence are important to experiencing optimal clinical outcomes.

\section{ACKNOWLEDGMENTS}

The authors would like to thank Jerry Kagan of Truven Health Analytics for programming and data analyses. Funding for this analysis was provided by Bristol-Myers Squibb and AstraZeneca. AstraZeneca funded the article processing charges associated with this publication. Parts of this manuscript were 
presented previously at the Academy of Managed Care Pharmacy 26th Annual Meeting \& Expo in Tampa, Florida, and the International Society for Pharmacoeconomics and Outcomes Research 19th Annual International Meeting in Montreal, Canada. All named authors meet the ICMJE criteria for authorship for this manuscript, take responsibility for the integrity of the work as a whole, and have given final approval to the version to be published.

Conflict of interest. Ms. Farr, Dr. Smith, and Mr. Johnston are employees of Truven Health Analytics, which was provided funding to conduct this study. Dr. Curkendall was an employee of Truven Health Analytics at the time the study was conducted. Drs. Sheehan and Kalsekar are employees of AstraZeneca, which funded this analysis.

Compliance with ethics guidelines. The analyses presented in this article are based on previously collected, de-identified data and do not contain any new studies with human subjects performed by any of the authors.

Open Access. This article is distributed under the terms of the Creative Commons Attribution Noncommercial License which permits any noncommercial use, distribution, and reproduction in any medium, provided the original author(s) and the source are credited.

\section{REFERENCES}

1. Centers for Disease Control and Prevention. National diabetes fact sheet: national estimates and general information on diabetes and prediabetes in the United States, 2011. http:// www.cdc.gov/diabetes/pubs/pdf/ndfs_2011.pdf. Accessed Jan 312014.

2. Inzucchi SE, Bergenstal RM, Buse JB, et al. Management of hyperglycemia in type 2 diabetes: a patient-centered approach: position statement of the American Diabetes Association (ADA) and the European Association for the Study of Diabetes (EASD). Diabetes Care. 2012;35:1364-79.

3. Garber AJ, Abrahamson MJ, Barzilay JI, et al. American Association of Clinical Endocrinologists' comprehensive diabetes management algorithm 2013 consensus statement. Endocr Pract. 2013;19:1-48.

4. Centers for Medicare and Medicaid Services. Part C and D Performance Data. http://www.cms.gov/ Medicare/Prescription-Drug-Coverage/Prescription DrugCovGenIn/PerformanceData.html. Accessed Mar 172014.

5. Asche C, LaFleur J, Conner C. A review of diabetes treatment adherence and the association with clinical and economic outcomes. Clin Ther. 2011;33:74-109.

6. Donnelly LA, Morris AD, Evans JM. DARTS/MEMO Collaboration. Adherence to insulin and its association with glycaemic control in patients with type 2 diabetes. QJM. 2007;100:345-50.

7. Ho PM, Rumsfeld JS, Masoudi FA, et al. Effect of medication nonadherence on hospitalization and mortality among patients with diabetes mellitus. Arch Intern Med. 2006;166:1836-41.

8. Rozenfeld Y, Hunt JS, Plauschinat C, Wong KS. Oral antidiabetic medication adherence and glycemic control in managed care. Am J Manag Care. $2008 ; 14: 71-5$.

9. Breitscheidel L, Stamenitis S, Dippel FW, Schoffski O. Economic impact of compliance to treatment with antidiabetes medication in type 2 diabetes mellitus: a review paper. J Med Econ. 2010;13:8-15.

10. Curkendall SM, Thomas N, Bell KF, Juneau PL, Weiss AJ. Predictors of medication adherence in patients with type 2 diabetes mellitus. Curr Med Res Opin. 2013;29:1275-86.

11. Roebuck MC, Liberman JN, Gemmill-Toyama M, Brennan TA. Medication adherence leads to lower health care use and costs despite increased drug spending. Health Aff (Millwood). 2011;30:91-9.

12. White TJ, Vanderplas A, Chang E, Dezii CM, Abrams GD. The costs of non-adherence to oral antihyperglycemic medication in individuals with diabetes mellitus and concomitant diabetes mellitus and cardiovascular disease in a managed care environment. Dis Manage Health Outcomes. 2004;12:181-8.

13. Jha AK, Aubert RE, Yao J, Teagarden JR, Epstein RS. Greater adherence to diabetes drugs is linked to less 
hospital use and could save nearly $\$ 5$ billion annually. Health Aff (Millwood). 2012;31:1836-46.

14. Cramer JA. A systematic review of adherence with medications for diabetes. Diabetes Care. 2004;27:1218-24.

15. Cramer JA, Benedict A, Muszbek N, Keskinaslan A, Khan ZM. The significance of compliance and persistence in the treatment of diabetes, hypertension, and dyslipidaemia: a revew. Int J Clin Pract. 2008;62:76-87.

16. Willliams JL, Walker RJ, Smalls BL, Campbell JA, Egede LE. Effective interventions to improve medication adherence in Type 2 diabetes: a systematic review. Diabetes Manag (Lond). 2014;4:29-48.

17. Malmenas M, Bouchard JR, Langer J. Retrospective real-world adherence in patients with type 2 diabetes initiating once-daily liraglutide $1.8 \mathrm{mg}$ or twice-daily exenatide $10 \mu \mathrm{g}$. Clin Ther. 2013;35:795-807.

18. Yang Y, Thumula V, Pace PF, Banahan BF 3rd, Wilkin NE, Lobb WB. Predictors of medication nonadherence among patients with diabetes in Medicare Part D programs: a retrospective cohort study. Clin Ther. 2009;31:2178-2188 (discussion 2150-2171).

19. Gregoire JP, Sirois C, Blanc G, Poirier P, Moisan J. Persistence patterns with oral antidiabetes drug treatment in newly treated patients: a populationbased study. Value Health. 2010;13:820-8.

20. Rathmann W, Kostev K, Gruenberger JB, Dworak M, Bader G, Giani G. Treatment persistence, hypoglycaemia and clinical outcomes in type 2 diabetes patients with dipeptidyl peptidase-4 inhibitors and sulphonylureas: a primary care database analysis. Diabetes Obes Metab. 2013;15:55-61.

21. Truven Health Analytics. MarketScan Bibliography. Available from: http://sites.truvenhealth.com/ bibliography/2014TruvenHealthMarketScanBibliogra phy.pdf.

22. Peterson AM, Nau DP, Cramer JA, Benner J, Gwadry-Sridhar F, Nichol M. A checklist for medication compliance and persistence studies using retrospective databases. Value Health. 2007;10:3-12.

23. Deyo RA, Cherkin DC, Ciol MA. Adapting a clinical comorbidity index for use with ICD-9-CM administrative databases. J Clin Epidemiol. 1992;45:613-9.

24. Gibson TB, Song X, Alemayehu B, et al. Cost sharing, adherence, and health outcomes in patients with diabetes. Am J Manag Care. 2010;16:589-600.

25. Inzucchi SE. Oral antihyperglycemic therapy for type 2 diabetes: scientific review. JAMA. 2002;287:360-72.

26. Malone M. Medications associated with weight gain. Ann Pharmacother. 2005;39:2046-55.

27. Betteridge DJ. Thiazolidinediones and fracture risk in patients with type 2 diabetes. Diabet Med. 2011;28:759-71.

28. Bristol-Myers Squib. Saxagliptin Prescribing Information. http://packageinserts.bms.com/pi/pi_ onglyza.pdf. Accessed Mar 172014.

29. Merck. Sitagliptin Prescribing Information. http:// www.merck.com/product/usa/pi_circulars/j/januvia/ januvia_pi.pdf. Accessed Mar 172014. 